\title{
Incidence and predictors of surgical site infections following caesarean sections at Bugando Medical Centre, Mwanza, Tanzania
}

Filbert J Mpogoro', Stephen E Mshana ${ }^{2 *}$, Mariam M Mirambo', Benson R Kidenya ${ }^{3}$, Balthazar Gumodoka ${ }^{1}$ and Can Imirzalioglu ${ }^{4}$

\begin{abstract}
Background: Surgical site infection (SSI) is the second most common infectious complication after urinary tract infection following a delivery by caesarean section (CS). At Bugando Medical Centre there has no study documenting the epidemiology of SSI after CS despite the large number of CSs performed and the relatively common occurrence of SSIs.
\end{abstract}

Methods: This was a prospective cohort study involving pregnant women who underwent a CS between October 2011 and February 2012 at Bugando Medical Centre. A total of 345 pregnant women were enrolled. Preoperative, intraoperative and postoperative data were collected using a standardized questionnaire. Wound specimens were collected and processed as per standard operative procedures; and susceptibility testing was carried out using a disc diffusion technique. Data was analyzed using STATA version 11.

Results: The overall cumulative incidence of SSI was 10.9\% with an incidence rate of 37.5 per 10,000 people/day (95\% Cl, 26.8-52.4). The median time from CS to the development of SSI was 7 days (interquartile range [IQR] $=6-9$ days). Six independent risk factors for post caesarean SSI as identified in this study by multivariate analysis are: hypertensive disorders of pregnancy (HR: 2.5; 95\% Cl, 1.1-5.6; $P=0.021$ ), severe anaemia (HR: 3.8; 95\% Cl, 1.2-12.4, $P=0.028)$, surgical wound class III (HR: 2.4; $95 \% \mathrm{Cl}, 1.1-5.0 ; P=0.021)$, multiple vaginal examinations (HR: $2.5 ; 95 \% \mathrm{Cl}$, $1.2-5.1 ; P=0.011$ ), prolonged duration of operation (HR: 2.6; $95 \% \mathrm{Cl}, 1.2-5.5 ; P=0.015)$ and an operation performed by an intern or junior doctor (HR: 4.0; $95 \% \mathrm{Cl}, 1.7-9.2 ; P=0.001$ ). Staphylococcus aureus was the most common organism (27.3\%), followed by Klebsiella pneumoniae (22.7\%). Patients with a SSI had a longer average hospital stay than those without a SSI $(12.7 \pm 6.9$ vs. $4 \pm 1.7 ; P<0.0001)$ and the case fatality rate among patients with a SSI was $2.9 \%$.

Conclusion: SSIs are common among women undergoing CSs at Bugando Medical Centre. SSIs were commonly associated with multiple factors. Strategies to control these factors are urgently needed to control SSIs post CS at Bugando Medical Centre and other centres in developing countries.

\section{Background}

Caesarean section (CS) is the most commonly performed major abdominal operations among women in both developed and developing countries [1]. Globally, the CS rate average is approximately $15 \%$ [1]; however there is a great variation in the rate of CSs in Latin

\footnotetext{
* Correspondence: mshana72@yahoo.com

${ }^{2}$ Department of Microbiology/Immunology Weill School of Medicine,

CUHAS-Bugando, BOX 1464, Mwanza, Tanzania

Full list of author information is available at the end of the article
}

America, from $1.6 \%$ in a Haitian hospital to $40 \%$ in Chile [2]. CS rates in Tanzania range from $21.4 \%-31.8 \%[3,4]$.

SSI is a common postoperative complication and is associated with significant morbidity and mortality [5-7]. The occurrence of an SSI following a CS reported in literature ranges from $0.3 \%$ in Turkey [7] to $24 \%$ [4] in Tanzania. The development of post CS infection depends on a complex interplay of many factors including: wound class, immune status, maternal age, hypertensive disorders, ASA classification, number of vaginal examinations, the virulence of the microorganisms, maternal

\section{Biomed Central}


weight, surgical techniques and premature rupture of membrane $[8,9]$. Pathogens that infect CS surgical wounds can be part of the patient's normal flora (endogenous source), originate from the skin, vaginal and peritoneal cavities, or can be acquired from the hospital environment, other infected patients, and surgeons (exogenous source) [10-12].

The occurrence, risk factors and distribution of the pathogens isolated from post CS SSIs at Bugando Medical Centre are not known. Moreover, there is no evidencebased data to support the preoperative and postoperative care protocols at the centre. Therefore this study was carried out to determine the incidence, susceptibility pattern of the isolates and factors associated with SSI among pregnant women undergoing CS at Bugando Medical Centre. The results of this study are necessary to develop an evidence-based treatment protocol for post CS SSIs in this clinical setting and other settings with similar problems.

\section{Methods and materials Study design}

This was a prospective cohort study to determine the incidence, predictors and patterns of surgical site infections among patients undergoing a CS at the Bugando Medical Centre(BMC) between October 2011 and February 2012. At $\mathrm{BMC}$, no routine operation theatre $(\mathrm{OT})$ and sterile services check up; however OT is undergoing major cleanliness once per week and whenever dirty case has been operated. All autoclaves are monitored using chemical and physical indicators daily.

\section{Inclusion and exclusion criteria}

All patients who delivered by CS at Bugando Medical Centre and consented for the study were included in the study. Patients who failed to provide information and those who died during the procedure or immediately after CS were excluded from the study.

\section{Sampling procedure and sample size}

Patients were serially enrolled until the sample size was reached. The sample size was calculated using the Schlesselman formula of the cohort study [13]. The occurrence rate used was the rate of SSIs among patients with obesity and those without obesity [14]. The minimum sample size obtained was 248, but the study enrolled 345 pregnant women.

\section{Data collection}

Data was collected using a standardized questionnaire. The data collected included patient, pre-/intra- and postoperative information such as demographic data, wound characteristics and laboratory investigations. Data collected included patient characteristics such as age, parity, co-morbidities such as BMI, DM, and hypertension; pre- operative data such as labour characteristics, preoperative hospital stay and preoperative skin preparation. Intraoperative data collected were type of caesarean section, duration of operation, extent of surgical wound contamination, surgical techniques, blood loss volume, antibiotic prophylaxis and type of anaesthesia used.

Surgical wounds were inspected at the time of dressing on day 3 then on day 7 when stitches are removed and thereafter telephone calls were used in every 4 days to enquire for development of SSI and all patients were reexamined at day 30 post operatively Surgical site infection was defined as per Centres for Disease Control and Prevention's National Nosocomial Infections Surveillance System as described below [15]. Superficial incisional SSI was defined as infection which occurs within 30 days after the operation and infection involves only skin or subcutaneous tissue of the incision and at least one of the following: Purulent drainage, with or without laboratory confirmation, from the superficial incision or organisms isolated from an aseptically obtained culture of fluid or tissue from the superficial incision; or at least one of the following signs or symptoms of infection: pain or tenderness, localized swelling, redness, or heat and superficial incision is deliberately opened by surgeon, unless incision is culture-negative, OR diagnosis of superficial incisional surgical site infection (SSI) by surgeon or attending physician.

Deep incisional SSI was defined as infection which occurs within 30 days after the operation and infection involves deep soft tissue (e.g. Fascial and muscle layers) of the incision and at least one of the following: Purulent drainage from the deep incision but not from the organ/ space component of the surgical site, OR a deep incision spontaneously dehisces or is deliberately opened by a surgeon when the patient has at least one of the following signs or symptoms: fever $(>38 \mathrm{C})$, localized pain, or tenderness, unless site is culture-negative, OR an abscess or other evidence of infection involving the deep incision is found on direct examination, during re-operation, or by histopathologic or radiological examination, OR diagnosis of a deep incisional SSI by a surgeon or attending physician.

Organ/space SSI infection was defined as infection occurs within 30 days after the operation and infection involves any part of the anatomy (e.g. organs or spaces), other than the incision, which was opened or manipulated during an operation and at least one of the following: Purulent drainage from a drain that is placed through a stab wound into the organ/space $O R$ organisms isolated from an aseptically obtained culture of fluid or tissue in the organ/space $O R$ an abscess or other evidence of infection involving the organ/space that is found on direct examination, during re-operation, or by histopathologic or radiological examination $O R$ diagnosis 
of an organ/space SSI by a surgeon or attending physician.

\section{Specimen collection and laboratory procedures}

Specimens were taken from all patients who clinically were suspected to have surgical site infections. Before sample collection surrounding skin was cleaned with $70 \%$ alcohol (Aldrich Sigma; Nairobi). Exudates were obtained from the open discharging wounds with a sterile cotton swab or with a syringe [16]. Swabs or aspirates were transported to the laboratory for processing immediately once obtained.

All specimens were processed in accordance with the standard operating procedures of the laboratory. Briefly specimens were inoculated on Blood agar and Mackonkey agar (Oxoid; UK) and incubated aerobically for 24-48 hours. Identification of bacteria was done using conventional physiological and biochemical methods. Biochemical and physiological methods included; gram stain, catalase reaction, coagulase reaction, haemolytic activity on 5\% sheep blood agar plate, hippurate hydrolysis and CAMP test for Gram positive bacteria while for gram negative; colonies morphology on blood agar and MacConkey agar, triple sugar iron (TSI) reaction, indole, motility, citrate, urease and hydrogen sulphide production (Oxoid, UK) were used $[17,18]$. Antimicrobial susceptibility of isolates was determined using disk diffusion method according to Clinical Laboratory standard Institute; for gram positive disks tested included penicillin G (10 IU), ampicillin $(10 \mu \mathrm{g})$, clindamycin $(15 \mu \mathrm{g})$, erythromycin $(15 \mu \mathrm{g})$, vancomycin $(30 \mu \mathrm{g})$, cefazolin $(30 \mu \mathrm{g})$ and ciprofloxacin $(5 \mu \mathrm{g})$ (Oxoid, UK). For gram negative disks tested included ampicillin $(10 \mu \mathrm{g})$, amoxicillin/clavunic acid $(20 / 10 \mu \mathrm{g})$, ciprofloxacin $(5 \mu \mathrm{g})$, gentamicin $(10 \mu \mathrm{g})$, ceftriaxone $(30 \mu \mathrm{g})$, ceftazidime $(30 \mu \mathrm{g})$ and meropenem $(10 \mu \mathrm{g})$ (Oxoid, UK). MRSA was identified using cefoxitin disc $(30 \mu \mathrm{g})$ and Oxacillin disc $(1 \mu \mathrm{g})$ and interpreted as per CLSI [19].

\section{Data analysis}

Data collected was entered into a computer using Epidata version 3.1 (CDC, Atlanta, USA) and analyzed using STATA version 11 (College Station, Texas, USA). Data was summarized in the form of proportions and frequent tables for categorical variables. Medians with standard deviation or medians with interquartile range were used to summarize continuous variables. A chisquare test was performed to test for significant associations between the predictor and outcome variables within the categorical variables and for continuous variables a Student t-test was performed. Hazard ratio (HR) with 95\% confidence interval (CI) was calculated to test for the strength of association between predictor variables and SSIs using univariate analysis followed by multivariate Cox regression analyses for all predictors found to be significant on the univariate analysis. Significant association was defined as a p-value of less than 0.05 .

\section{Ethical clearance}

Catholic University of Health and Allied Sciences /Bugando Medical Centre review board cleared the study and informed consents were obtained from patients.

\section{Study limitations}

Twenty six percent of the patients who developed an SSI after discharge did not return for specimen collection. No anaerobic culture was done and lack of antibiotic policy may have affected the SSI rate.

\section{Results}

Patients' characteristics

A total of 3250 deliveries were performed during the study period, $2476(76.2 \%)$ by the vaginal route and 774 (23.8\%) by CS (Figure 1). A total of 345 pregnant women who underwent CSs were enrolled and followed up for 30 days for development of SSI. Of these 345 CS; 319 $(92.5 \%)$ were emergency procedures and $26(7.5 \%)$ as elective procedures (Table 1).

The age of the women ranged from 14 to 44 years with a mean of $26.8 \pm 5.8$ years. The majority of women $(273,79.13 \%)$ were between 20 to 34 years of age and 218 (63.3\%) were multiparas. The gestational age at caesarean delivery ranged between 28-44 weeks and a total of 268 neonates $(79.8 \%)$ were delivered at term. Scars as indication of previous CS were found in 106 (30.7\%) patients (Table 2).

\section{Magnitude and burden of surgical site infections}

During the study period, 34 patients developed SSIs resulting in an overall cumulative incidence of 34/312 (10.9\%) and an incidence rate of 37.5 per 10,000 people/ day (95\% CI, 26.8-52.4). Types of SSI found were superficial SSI $(21,61.8 \%)$, organ space $(8,23.5 \%)$ and deep SSI (5, 14.7\%). All SSIs occurred among patients who had emergency CSs. The rate of SSI was $12 / 202(5.9 \%)$ for clean wounds and 22/143(29.4\%) for clean-contaminated wounds ( $\mathrm{p}=0.028$ Fischer exact test). Most SSIs occurred among patients who had a vertical skin incision (31/43; 91.2\%).

All SSIs occurred between the 3rd and 22nd day postoperatively with a median time of occurrence of 7 days post operation (IQR 6-9 days). Patients with a SSI had longer hospital stays than those without a SSI $(12.7 \pm 6.9$ vs. $4 \pm 1.7 ; P<0.0001$ ). The majority of patients with SSIs $(n=23 / 67.6 \%)$ were readmitted and one patient with an organ/space SSI died 2 days post relaparotomy due to septicemia. 


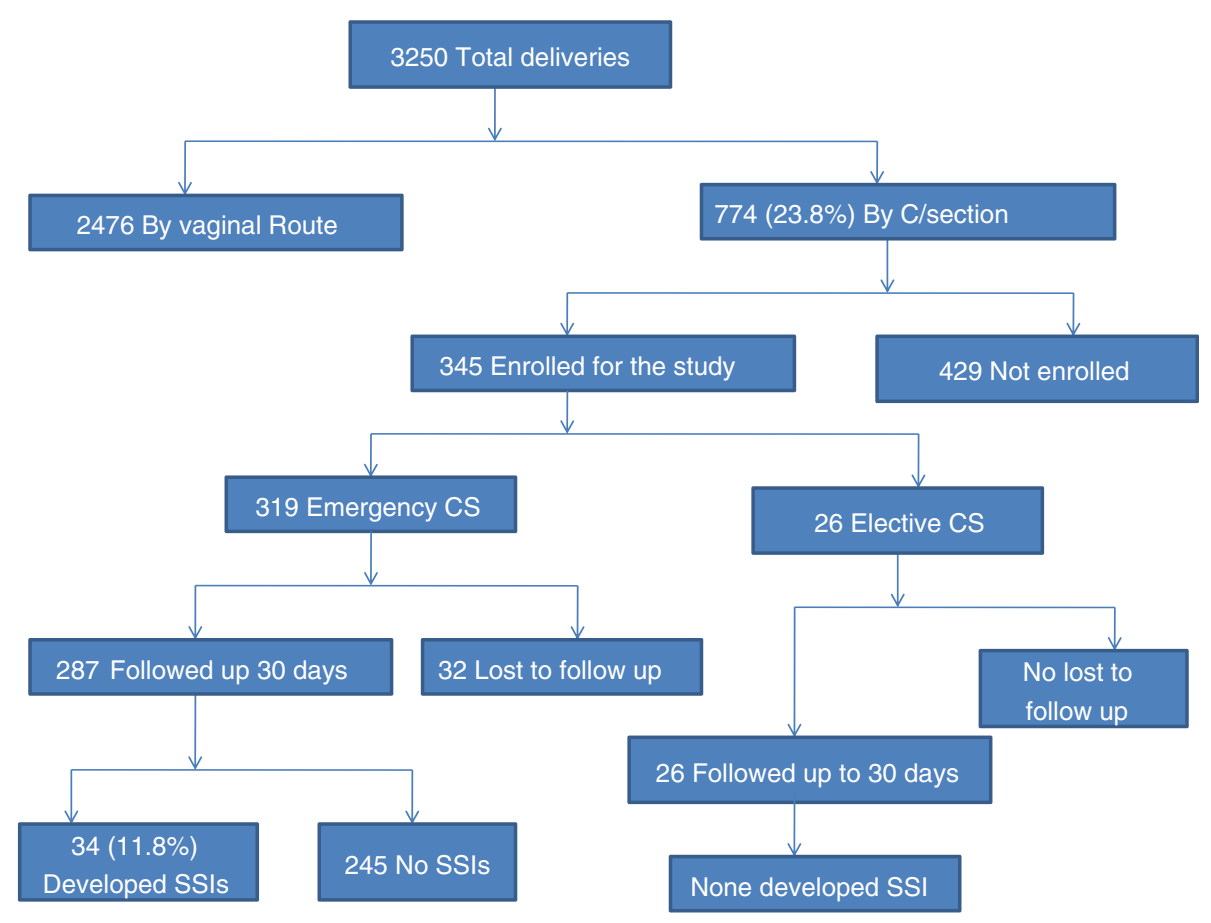

Figure 1 Flow chart of patient recruitment and follow up.

\section{Predictors of surgical site infections}

Univariate analysis indicated that post caesarean SSI occurred significantly more often among women with: hypertensive disorders of pregnancy $(\mathrm{HR}=2.2 ; 95 \% \mathrm{CI}=1.1-4.7$; $p=0.031)$, higher ASA physical status class $(\mathrm{HR}=2.7$; $95 \% \mathrm{CI}=1.3-5.5 ; p=0.005)$, prolonged duration of labor $(\mathrm{HR}=3.0 ; 95 \% \mathrm{CI}=1.5-6.0 ; p=0.002)$, rupture of membranes prior to surgery lasting 8 hours or longer, $(\mathrm{HR}=2.7$; $95 \% \mathrm{CI}=1.3-5.8 ; p=0.011), 3$ or more vaginal examinations $(\mathrm{HR}=3.3 ; 95 \% \mathrm{CI}=1.7-6.5 ; p=0.001)$, higher wound class prior to surgery $(\mathrm{HR}=2.7 ; 95 \% \mathrm{CI}=1.4-5.5$; $P=0.005)$, vertical skin incision $(\mathrm{HR}=3.6 ; 95 \% \mathrm{CI}=1.1$ $11.8 ; p=0.034$ ), prolonged duration of operation (a surgical procedure lasting longer than 1 hour $)(\mathrm{HR}=2.4 ; 95 \%$ $\mathrm{CI}=1.1-5.0 ; p=0.020)$, and a procedure performed by an intern or junior doctor $(\mathrm{HR}=2.8 ; 95 \% \mathrm{CI}=1.3-6.1$; $p=0.012$ ) (Tables 3 and 4).

Approximately $92 \%$ of CSs in this study were emergency procedures and all SSIs occurred in this group. However the following factors had no effect on the occurrence of SSI; age, gravidity, parity, gestational age at delivery, obesity, diabetes mellitus, anemia, preoperative length of stay, HIV infection, type of anaesthesia used, number of people in theatre, timing of antibiotic prophylaxis and the amount of blood loss intraoperatively.

Multivariate analysis did not include factors with high collinearity such as ASA and duration of rupture of membrane. The independent risk factors for post caesarean SSI were identified in this study by multivariate
Cox regression analysis were hypertensive disorders of pregnancy $(\mathrm{HR}=2.9 ; 95 \% \mathrm{CI}, 1.4-6.4 ; p=0.006)$; contaminated wound $(\mathrm{HR}=2.5 ; 95 \% \mathrm{CI}, 1.2-5.1 ; p=0.016)$, multiple vaginal examinations $(\mathrm{HR}=2.6$; $95 \% \mathrm{CI}, 1.3-5.3$; $p=0.008)$, prolonged duration of operation $(\mathrm{HR}=2.3$; 95\% CI,1.1-4.8; $p=0.001)$ and an operation performed by an intern or junior doctor $(\mathrm{HR}=4.2$; $95 \% \mathrm{CI}, 1.8-9.5$; $p=0.030$ ) (Table 5).

In this study, the risk of developing a SSI increased significantly from a HR of 2.9 for patients with a National Nosocomial Infections Surveillance System (NNIS) risk index of 1 to a HR of 9.1 in patients with a NNIS System risk index of 2 .

Almost all (344, 99.7\%) women received antibiotics as prophylaxis with different timings of administration either before or after skin incision. No specific policy was followed, and the choice of antibiotics used was based on indication of CS and surgeons preference. The antibiotics given could be divided into ampicillin based regimens (170/344) and non-ampicillin based regimen combinations (174/344). A higher SSI rate was observed among patients which received the ampicillin based regimen $(11.5 \%)$ compared with those which received nonampicillin based regimen (8.2\%, $\mathrm{p}=0.304$, HR 1.4).

\section{Bacterial isolates and susceptibility pattern}

Pus swabs for aerobic culture and sensitivity were collected for 25 (73.5\%) of the clinically suspected postoperative infection cases. Of 25 aerobic cultures, 18 (72.0\%) 
Table 1 Clinical characteristics of patients undergoing caesarean sections, with and without subsequent surgical site infections

\begin{tabular}{lr}
\hline Characteristic & $\mathbf{n}(\%)$ \\
\hline $\begin{array}{ll}\text { Age in years } \\
\quad<20\end{array}$ & $30(8.7)$ \\
$20-34$ & $273(79.1)$ \\
$\geq 35$ & $42(12.2)$ \\
Parity & \\
$\quad$ Nulliparous & $127(36.8)$ \\
$1-3$ & $180(52.2)$ \\
$\geq 4$ & $38(11.0)$
\end{tabular}

Gestational age in weeks

37

$\geq 37$

Mode of admission

Self

Referral

Onset of labor

Spontaneous

Induced

No labor

Type of skin incision

Vertical

Transverse

Type of caesarean section

Elective

Emergency

Type of skin incision

Transverse

Vertical

Number of prior caesarean sections

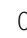

2

3

4

\section{HIV status}

Positive

Negative

Unknown

Body mass index $(\mathrm{N}=345)$

Underweight $(<18.5)$

Normal weight (18.5-24.9)

Overweight (25-29.9)

Obesity $\geq 30$

$136(39.4)$
Table 2 Indications for caesarean section

\begin{tabular}{lr}
\hline Indication & N (\%) \\
\hline Previous caesarean delivery & $106(30.7)$ \\
Fetal distress & $54(15.7)$ \\
Prolonged labor & $48(13.9)$ \\
Malpresentation & $32(9.3)$ \\
Obstructed labor & $28(8.1)$ \\
Hypertensive disorders of pregnancy & $30(8.7)$ \\
Antepartum hemorrhage & $9(2.6)$ \\
Premature rupture of membranes & $7(2.0)$ \\
Others* & $31(9.0)$ \\
Total & $\mathbf{3 4 5}$ (100.00) \\
\hline *Included maternal distress, maternal request, big baby, twin with & \\
Malpresentation, ruptured uterus, cord accident and failed induction of labor.
\end{tabular}

were culture positive and 4 had significant polymicrobial infections, resulting in a total of 22 bacterial isolates.

Among the etiological agents isolated, 14 (63.6\%) were gram-negative bacilli. Staphylococcus aureus was the most common organism (6, 27.3\%). Of Staphylococcus aureus, 5 (83.3\%) were methicillin sensitive Staphylococcus aureus (MSSA) and 1 (16.7\%) was methicillin resistant Staphylococcus aureus (MRSA). Other isolates include Klebsiella spp (5, 22.7\%), Escherichia coli (3, 13.6\%), Acinetobacter spp (2, 9.1\%), Pseudomonas spp (2, 9.1\%), Proteus spp (1, 4.5\%), Enterobacter spp (1, 4.5\%), Micrococcus spp (1, 4.5\%) and coagulase negative Staphylococcus spp (1, 4.5\%).

The majority of gram-positive bacteria isolated were
$26(7.5)$

$319(92.5)$

$86(24.9)$

259 (75.1)

$214(62.0)$

88 (25.5)

$31(9.0)$

$10(2.9)$

2 (0.6)

22 (6.4)

218 (63.2)

105 (30.4)

$1(0.3)$

46 (13.3)

$162(45.0)$ $100 \%$ sensitive to vancomycin and imipenem and $85 \%$ sensitive to cefazolin. Most of the enteric gram-negative bacteria were highly resistant to ampicillin (100\%), amoxicillin/clavulanate (93\%), sulphamethaxazole/trimethoprim (78.5\%), fosfomycin (70\%), tetracycline (70\%) and gentamicin (35.7\%). They were 100\%, 85.7\%, 85.7\% and $78 \%$ sensitive to meropenem, ceftazidime, ciprofloxacin and ceftriaxone respectively. A total of 2 (13\%) of the enteric gram-negative bacteria were found to produce extended spectrum beta lactamases (ESBL). Out of 8 organ infections, $5(62.5 \%)$ were due to gram-negative enteric bacteria and $29.4 \%(5 / 17)$ of incisional SSIs were caused by Staphylococcus aureus.

\section{Discussion}

Magnitude of post cesarean section surgical site infection As reported in many other studies in the developing countries [20,21],the incidence of SSIs in the present study was higher than in developed countries. This could be explained by standard of hygiene practiced in developed countries. In this study the incidence of SSIs following CS was $10.9 \%$, which is lower than previously reported in Tanzania. At study at St. Francis Ifakara Hospital reported a cumulative incidence rate of $24 \%$ 
Table 3 Univariate Analysis of preoperative risk factors for surgical site infection (SSI) in patients who underwent caesarean sections

\begin{tabular}{|c|c|c|c|c|}
\hline \multirow[b]{2}{*}{ Predictive factors } & \multicolumn{2}{|c|}{ Surgical site infection (SSI) } & \multirow[b]{2}{*}{ HR $(95 \% \mathrm{Cl})$} & \multirow[b]{2}{*}{$p$ - value } \\
\hline & Yes n (\%) & No $\mathrm{n}(\%)$ & & \\
\hline \multicolumn{5}{|l|}{ Age in years } \\
\hline$>30$ & $4(4.4)$ & $86(95.60$ & 1.0 & \\
\hline$\leq 30$ & $30(11.8)$ & $225(88.2)$ & $2.8(1.0-7.9)$ & 0.055 \\
\hline \multicolumn{5}{|l|}{ Parity } \\
\hline Multiparas & $19(8.7)$ & $119(91.3)$ & 1.0 & \\
\hline Nulliparous & $15(11.8)$ & $112(88.2)$ & $1.3(0.7-2.6)$ & 0.393 \\
\hline \multicolumn{5}{|l|}{ BMl $\neq$} \\
\hline None obese & $30(9.6)$ & $282(90.4)$ & 1.0 & \\
\hline Obese & $4(12.1)$ & 29 (87.9) & $1.3(0.4-3.6)$ & 0.647 \\
\hline \multicolumn{5}{|l|}{ HIV status } \\
\hline Negative & $23(10.6)$ & $195(89.5)$ & 1.0 & \\
\hline Positive & $4(18.2)$ & $18(81.8)$ & $1.8(0.6-5.1)$ & 0.295 \\
\hline \multicolumn{5}{|l|}{ Hypertension } \\
\hline No & $24(8.3)$ & $265(91.7)$ & 1.0 & \\
\hline Yes & $10(17.9)$ & $46(82.1)$ & $2.2(1.1-4.7)$ & 0.031 \\
\hline \multicolumn{5}{|l|}{ ASA score* } \\
\hline 1 or 2 & $22(7.8)$ & $260(92.2)$ & 1.0 & \\
\hline$\geq 3$ & $12(19.1)$ & $51(80.9)$ & $2.7(1.3-5.5)$ & 0.005 \\
\hline \multicolumn{5}{|c|}{ Hemoglobin level in $\mathrm{g} / \mathrm{dl}$} \\
\hline$>11$ & $24(10.0)$ & $217(90.0)$ & 1.0 & \\
\hline$<11$ & $10(10.4)$ & $86(89.6)$ & $1.0(0.5-2.1)$ & 0.960 \\
\hline \multicolumn{5}{|c|}{ Duration of ROMt in hrs } \\
\hline$\leq 8$ & $25(8.3)$ & $277(91.7)$ & 1.0 & \\
\hline$>8$ & $9(20.9)$ & $34(79.1)$ & $2.7(1.3-5.8)$ & 0.011 \\
\hline \multicolumn{5}{|c|}{ Number of vaginal exams } \\
\hline$\leq 3$ & $20(7.1)$ & $260(92.9)$ & 1.0 & \\
\hline$>4$ & $14(21.5)$ & $51(78.5)$ & $3.3(1.7-6.5)$ & 0.001 \\
\hline \multicolumn{5}{|c|}{ Duration of labor in hrs } \\
\hline$\leq 12$ & $20(7.3)$ & $256(92.7)$ & 1.0 & \\
\hline$>12$ & $14(20.3)$ & $55(79.7)$ & $3.0(1.5-6.0)$ & 0.002 \\
\hline \multicolumn{5}{|c|}{ Preop length of stays in hrs } \\
\hline$\leq 12$ & $17(7.8)$ & $200(92.2)$ & 1.0 & \\
\hline$>12$ & $17(13.3)$ & $111(86.7)$ & $1.7(0.9-3.4)$ & 0.116 \\
\hline
\end{tabular}

*ASA $=$ American Society of Anesthesiologists $+\mathrm{ROM}=$ rupture of membranes. $\neq \mathrm{BMI}=$ Body mass index in $\mathrm{kg} / \mathrm{M}^{2}$.

[20], which could be explained by lack of infection prevention policies in Ifakara during their study. The incidence of SSI following CSs was found to vary widely depending on the surveillance methods used to identify infections, criteria used to define SSI, postoperative hospital stays, antibiotic prophylaxis and the patient population $[20,21]$. In the present study the majority of patients had clean contaminated wounds. Previous studies have reported that patients with contaminated wounds had a 2.7 fold increased risk of developing a SSI than those with clean contaminated wounds [22-24]. The majority of post CS SSIs found in this study were superficial infections followed by deep tissue infections, similar to the findings were observed in previous studies [25]. Also 
Table 4 Univariate analysis of intraoperative risk factors for surgical site infection (SSI) among patients who underwent caesarean sections

\begin{tabular}{|c|c|c|c|c|}
\hline \multirow[b]{2}{*}{ Predictive factors } & \multicolumn{2}{|c|}{ Surgical site infection (SSI) } & \multirow[b]{2}{*}{$\mathrm{HR}(95 \% \mathrm{Cl})$} & \multirow[b]{2}{*}{$p$ - value } \\
\hline & Yes n (\%) & No $n(\%)$ & & \\
\hline \multicolumn{5}{|l|}{ Wound class } \\
\hline Clean or clean-contaminated & $12(5.9)$ & $190(94.1)$ & 1.0 & \\
\hline Contaminated or dirty & $22(15.4)$ & $121(84.6)$ & $2.7(1.4-5.5)$ & 0.005 \\
\hline \multicolumn{5}{|l|}{ Type of anaesthesia used } \\
\hline Regional & $32(9.7)$ & $298(90.30$ & 1.0 & \\
\hline General & $2(13.3)$ & $13(86.7)$ & $1.5(0.4-6.5)$ & 0.551 \\
\hline \multicolumn{5}{|l|}{ Type of skin incision } \\
\hline Transverse & $3(3.5)$ & $83(96.5)$ & 1.0 & \\
\hline Vertical & $31(12.0)$ & $228(88.0)$ & $3.6(1.1-11.8)$ & 0.034 \\
\hline \multicolumn{5}{|l|}{ Duration of procedure in mins } \\
\hline$\leq 60$ & $24(8.3)$ & $265(91.7)$ & 1.0 & \\
\hline$>60$ & $10(17.9)$ & $46(82.1)$ & $2.4(1.1-5.0)$ & 0.020 \\
\hline \multicolumn{5}{|l|}{ Type of surgeon } \\
\hline Senior* & $26(8.4)$ & $282(91.6)$ & 1.0 & \\
\hline Junior** & $8(21.6)$ & $29(78.4)$ & $2.8(1.3-6.1)$ & 0.012 \\
\hline \multicolumn{5}{|l|}{ Number of people in theatre } \\
\hline$\leq 5$ & $8(13.8)$ & $50(86.2)$ & 1.0 & \\
\hline$>5$ & $26(9.1)$ & $260(90.9)$ & $0.7(0.3-1.4)$ & 0.289 \\
\hline \multicolumn{5}{|l|}{ Estimated blood loss in mls } \\
\hline$\leq 500$ & $25(9.3)$ & $245(90.7)$ & 1.0 & \\
\hline$>500$ & $9(12.0)$ & $66(88.0)$ & $1.4(0.6-2.9)$ & 0.436 \\
\hline \multicolumn{5}{|l|}{ Antibiotic used } \\
\hline Non-ampicillin regimen & $14(8.2)$ & $156(91.8)$ & 1.0 & \\
\hline Ampicillin regimen & $20(11.5)$ & $154(88.5)$ & $1.4(0.7-2.8)$ & 0.304 \\
\hline \multicolumn{5}{|l|}{ Duration of antibiotic course } \\
\hline Single dose & $7(8.5)$ & $75(91.5)$ & $0.8(0.4-1.8)$ & 0.608 \\
\hline Multiple doses & $27(10.3)$ & $235(89.7)$ & 1.0 & \\
\hline
\end{tabular}

Abbreviations: $H R$ hazard ratio, $\mathrm{Cl}$ confidence interval.

*Senior doctor included postgraduate student, registered medical doctor and specialist.

**Junior surgeon defined an intern doctor.

Table 5 Multivariate Model of risk factors for surgical site infection (SSI) in patients who underwent caesarean sections

\begin{tabular}{lcc}
\hline Independent risk factors & HR $(\mathbf{9 5} \% \mathrm{Cl})$ & $\boldsymbol{P}$ - value \\
\hline Hypertensive disorders of pregnancy & $2.9(1.4-6.4)$ & 0.006 \\
Contaminated wound & $2.5(1.2-5.1)$ & 0.016 \\
Multiple vaginal examinations & $2.6(1.3-5.3)$ & 0.008 \\
Operation done by Intern doctor & $4.2(1.8-9.5)$ & 0.001 \\
Severe anaemia $(\mathrm{Hb}<7 \mathrm{~g} / \mathrm{dl})$ & $3.8(1.2-12.4)$ & 0.028 \\
Duration of procedure $>60$ minutes & $2.3(1.1-4.8)$ & 0.030 \\
\hline
\end{tabular}

$\mathrm{HR}=$ hazard ratio, $\mathrm{Cl}=$ confidence. reported previously [26], the incidence of deep tissue infections in this study was $14.7 \%$ and that of organ space infection was $5 \%$.

Enteric gram-negative bacteria have previously been reported to be associated with severe SSI $[27,28]$. In the present study $62.5 \%$ of organ space infections were caused by gram negative enteric bacteria. This could be explained by the synergistic effect of facultative anaerobes and anaerobic infections.

Predictors of post cesarean section surgical site infection Various risk factors have been found to predict post caesarean SSI $[8,9,26]$. One patient factor is younger maternal age $[14,29]$; in the present study, an association was recorded between maternal age and SSI, suggesting that 
women aged 30 years or younger were more likely to have a SSI than those older than 30 years. Despite this trend, the association was not proven to be statistically significant $(\mathrm{p}=0.055)$. In accordance with previous studies $[24,30]$, hypertensive disorder of pregnancy was a predictor of SSI when analysed by both univariate $(\mathrm{p}=0.031)$ and multivariate analysis $(\mathrm{p}=0.006)$. This link could be explained by the chronic alteration of peripheral blood supply due to the increased vascular resistance.

In contrast to previous studies [14,29], the association of obesity and SSI was not statistically significant ( $\mathrm{p}=0.647$, HR 1.3). Obesity has previously been reported to predict SSI via various possible factors, including the relative avascularity of adipose tissue. Another factor may be technical difficulties of handling adipose tissue which can result in more traumas to the anterior abdominal wall, or difficulty in obliterating dead space in the fat-tissue of the abdominal wall. The lack of significant association in this study may be due to the fact the Body Mass Index of patients in this study was measured during labour rather than before pregnancy.

ASA physical status classification score of 3 or more was significantly found to predict SSI $(p=0.005)$, in agreement with previously reported findings [31,32]. Prolonged labor ( $\geq 12 \mathrm{hrs}$ ), prolonged rupture of membranes $(\geq 8 \mathrm{hrs})$ and multiple vaginal examinations $(\geq 4$ times) were significant predictors of SSI in this study, in agreement with previously obtained results $[4,8,26]$. Normally during pregnancy, cervical mucus plug, fetal membranes and amniotic fluid all serve as barriers to infection. However when the membrane is ruptured, this protective effect is gradually reduced over time as amniotic fluid becomes no longer sterile. It is thought that the non-sterile amniotic fluid may act as a transport medium by which bacteria come into contact with the uterine and skin incisions leading to chorioamnionitis and its sequelae.

Another important risk factor for SSI is the absence or delay of antibiotic prophylaxis. In this study there was no standard policy of antibiotic prescription. The choice of antibiotic administered depends on the surgeon and indication of CS. The antibiotics given in this study could be divided into two groups; an ampicillin based regimen and a non-ampicillin based regimen. The timing of antibiotic administration was not consistent. In contrast to previous studies $[33,34]$ no significant difference was observed regarding type of antibiotic prophylaxis and SSI.

In this study emergency procedures were a strong predictor of SSI. This finding could be due to sampling bias because approximately $92.5 \%$ of CSs were done on an emergence basis. Vertical incision was significantly found to predict SSI; women with vertical skin incisions had a 3.6 fold risk of developing a SSI compared to those with transverse skin incision, and this has been observed before [29]. Prolonged operating times of longer than one hour has previously been found to be associated with SSI due to increased duration to exposure to microorganisms in the operating theatre $[16,26,35]$ and this was confirmed by this study. The experience of surgeons performing the CS was also a critical determinant of SSI. Excellent surgical technique such as effective homeostasis while preserving adequate blood supply, preventing hypothermia, gently handling tissues, avoiding inadvertent entries into a hollow viscus, choice of appropriate suture material, eradicating dead space, and appropriately managing the postoperative incision are widely believed to reduce the risk of SSI [36]. In the present study, operations performed by an intern or junior surgeon increased the risk for SSI 4 fold, as shown by multivariate analysis. This could be explained by the fact that majority of junior surgeons made vertical incisions, had less experience in handling the tissue and control of blood loss, and the procedures were prolonged for more than 1 hour.

\section{Bacterial infection pattern}

The microbial etiology of post CS SSIs has been shown to be diverse, being associated with both vaginal microorganisms such as Escherichia coli, group B streptococcus (GBS) and Enterococcus spp, or with nasopharyngeal flora such as Staphylococcus aureus or skin flora such Staphylococcus epidermidis [16]. Staphylococcus aureus has been found to be the most common cause of SSI post CS [17,37]. Other organisms such as Escherichia coli, Klebsiella spp., Pseudomonas aeruginosa, Enterobacter spp., Proteus spp. and Enterococcus spp., show a variable distribution pattern [16]. A similar pattern has been observed in this study; Staphylococcus aureus was the most common isolate taken from superficial skin infections followed by Klebsiella pneumoniae in deep and organ infections. As described in other studies most of Staphylococcus aureus isolated from SSIs were MSSA and only 1 isolate (16.7\%) was found to be MRSA. The prevalence of MRSA is similar to the previous study carried out in the same hospital in 2009 [20]. In the present study the majority (37.5\%) of Staphylococcus aureus isolates were resistant to ampicillin, co-trimoxazole and erythromycin; similar to the findings of Fantahamu et al. [38]. The rate of resistance to these antibiotics was higher than the rate of resistance to ciprofloxacin and gentamicin, which could be explained by self prescription to these antibiotics in the community.

As reported previously, the majority of gram-negative enteric bacteria were highly resistant to ampicillin, amoxycillin/clavulanate, tetracycline and co-trimoxazole [11]. A total of $10-22 \%$ of gram-negative enteric bacteria was found to be resistant to third generation cephalosporins, in accordance with previous findings [11]. In 
this study only one patient with organ space infection died, and this was due to a polymicrobial infection of Staphylococcus aureus and Klebsiella pneumoniae which was an ESBL producer. Patients with a SSI had prolonged hospitalization compared with those without a SSI (12.7 vs 4.1 days), similar to the results observed by Killian et al. [26].

\section{Conclusion}

Hypertensive disorders of pregnancy, contaminated wound, multiple vaginal examinations, operations carried out by an intern or junior doctor and prolonged duration of the surgical procedure (longer than 60 minutes) have been found to be independent factors which increase the risk of SSI at Bugando Medical Centre. Identifying high-risk patients who require intensive postoperative care is critical in order to reduce the incidence of SSIs. This can be achieved if independent risk factors for SSI are well understood within the given clinical setting.

\section{Competing interests}

The authors declare that they have no competing interests.

\begin{abstract}
Authors' contributions
FJM; participated in collecting specimens, collecting clinical data and follow up of the patients and data analysis, SEM; participated in the design and execution of the work, performed microbiological procedures, data analysis, interpretation of data and preparation of the manuscript, MM: Perform data analysis and writing the manuscript; BRK; participated in data analysis, BG; designed the study, collected clinical data and participated in manuscript writing, Cl; Designed the study, participated in the interpretation of the data and manuscript writing. All authors have read and approved the final manuscript.
\end{abstract}

\section{Acknowledgements}

The authors would like to acknowledge the technical support provided by the members of the Departments of Microbiology/lmmunology and Obstetric and Gyanecology. We thank Mary Louise Shushu, Hezron Bassu and Benard Okamo for their excellent technical assistance.

\section{Author details}

'Department of Obstetrics and Gynecology Weill School of Medicine, CUHAS-Bugando, Mwanza, Tanzania. ²Department of Microbiology/ Immunology Weill School of Medicine, CUHAS-Bugando, BOX 1464, Mwanza, Tanzania. ${ }^{3}$ Department of Biochemistry and Molecular Biology, Weill School of Medicine, CUHAS-Bugando, Mwanza, Tanzania. ${ }^{4}$ Institute of Medical Microbiology, Justus-Liebig-University of Giessen, Giessen, Germany.

Received: 12 December 2012 Accepted: 5 August 2014

Published: 11 August 2014

\section{References}

1. Betrán AP, Merialdi M, Lauer JA, Bing-Shun W, Thomas J, Van Look P Wagner M: Rates of caesarean section: analysis of global, regional and national estimates. Paediatr Perinat Epidemiol 2007, 21(2):98-113.

2. Dumont $A$, De Bernis L, Bouvier-olle M-H, Bréart G: Caesarean section rate for maternal indication in sub-Saharan Africa: a systematic review. Lancet 2001, 358(9290):1328-1333.

3. Sørbye IK, Vangen S, Oneko O, Sundby J, Bergsjø P: Caesarean section among referred and self-referred birthing women: a cohort study from a tertiary hospital, northeastern Tanzania. BMC Pregnancy Childbirth 2011, 11(1):55.

4. Muganyizi $P$, Kidanto $H$, Kazaura M, Massawe S: Caesarean section: trend and associated factors in Tanzania. Afr J Midwifery Womens Health 2008, 2(2):65-68.
5. Kirkland KB, Briggs JP, Trivette SL, Wilkinson WE, Sexton DJ: The impact of surgical-site infections in the 1990s: attributable mortality, excess length of hospitalization, and extra costs. Infect Control Hosp Epidemiol 1999, 20(11):725-730.

6. De Lissovoy G, Fraeman K, Hutchins V, Murphy D, Song D, Vaughn BB: Surgical site infection: incidence and impact on hospital utilization and treatment costs. Am J Infect Control 2009, 37(5):387-397.

7. Tran TS, Jamulitrat S, Chongsuvivatwong V, Geater A: Risk factors for postcesarean surgical site infection. Obstet Gynecol 2000, 95(3):367-371.

8. Olsen MA, Butler AM, Willers DM, Devkota P, Gross GA, Fraser VJ: Risk factors for surgical site infection after low transverse cesarean section. Risk 2008, 29(6):477-484.

9. Gong SP, Guo HX, Zhou HZ, Chen L, Yu YH: Morbidity and risk factors for surgical site infection following cesarean section in Guangdong Province, China. J Obstet Gynaecol Res 2012, 38(3):509-515.

10. System NNIS: National Nosocomial Infections Surveillance (NNIS) System Report, data summary from January 1992 through June 2004, issued October 2004. Am J Infect Control 2004, 32(8):470.

11. Anguzu J, Olila D: Drug sensitivity patterns of bacterial isolates from septic post-operative wounds in a regional referral hospital in Uganda. Afr Health Sci 2007, 7(3):148-154.

12. Giacometti A, Cirioni O, Schimizzi A, Del Prete M, Barchiesi F, D'errico M, Petrelli E, Scalise G: Epidemiology and microbiology of surgical wound infections. J Clin Microbiol 2000, 38(2):918-922.

13. Schlesselman JJ: Sample size requirements in cohort and case-control studies of disease. Am J Epidemiol 1974, 99(6):381-384.

14. Myles TD, Gooch J, Santolaya J: Obesity as an independent risk factor for infectious morbidity in patients who undergo cesarean delivery. Obstet Gynecol 2002, 100(5, Part 1):959-964.

15. Horan TC, Gaynes RP, Martone WJ, Jarvis WR, Grace Emori T: CDC definitions of nosocomial surgical site infections, 1992: a modification of CDC definitions of surgical wound infections. Am J Infect Control 1992, 20(5):271-274.

16. Kaplan N, Smadi A, Al-Taani M, El-Qudah M: Microbiology of wound infection after caesarean section in a Jordanian hospital. East Mediterr Health J 2003, 9(5/6):1069.

17. Mawalla B, Mshana SE, Chalya PL, Imirzalioglu C, Mahalu W: Predictors of surgical site infections among patients undergoing major surgery at Bugando Medical Centre in Northwestern Tanzania. BMC Surg 2011, 11(1):21.

18. Mshana SE, Kamugisha E, Mirambo M, Chakraborty T, Lyamuya EF: Prevalence of multiresistant gram-negative organisms in a tertiary hospital in Mwanza, Tanzania. BMC Res Notes 2009, 2(1):49.

19. Wikler MA, Cockerill FR: Clinical and Laboratory Standards Institute. In Performance Standards for Antimicrobial Susceptibility Testing: Eighteenth Informational Supplement Wayne: Clinical and Laboratory Standards Institute. 2008.

20. Fehr J, Hatz C, Soka I, Kibatala P, Urassa H, Smith T, Mshinda H, Frei R, Widmer A: Risk factors for surgical site infection in a Tanzanian district hospital: a challenge for the traditional National Nosocomial Infections Surveillance system index. Risk 2006, 27(12):1401-1404.

21. Mitt $P$, Lang K, Peri A, Maimets $M$ : Surgical-site infections following cesarean section in an Estonian university hospital: postdischarge surveillance and analysis of risk factors. Infect Control Hosp Epidemiol 2005, 26(5):449-454.

22. Henderson $E$, Love $E$ : Incidence of hospital-acquired infections associated with caesarean section. J Hosp Infect 1995, 29(4):245-255.

23. Beattie $P$, Rings $T$, Hunter $M$, Lake $Y$ : Risk factors for wound infection following caesarean section. Aust N Z J Obstet Gynaecol 1994, 34(4):398-402.

24. Schneid-Kofman N, Sheiner E, Levy A, Holcberg G: Risk factors for wound infection following cesarean deliveries. Int J Gynecol Obstet 2005, 90(1):10-15

25. Chia J, Tan K, Tay L: A survey of postoperative wound infections in obstetrics and gynaecology-the Kandang Kerbau Hospital experience. Singap Med J 1993, 34(3):221-224.

26. Killian CA, Graffunder EM, Vinciguerra TJ, Venezia RA: Risk factors for surgical-site infections following cesarean section. Infect Control Hosp Epidemiol 2001, 22(10):613-617.

27. Weinstein RA, Gaynes R, Edwards JR: Overview of nosocomial infections caused by gram-negative bacilli. Clin Infect Dis 2005, 41(6):848-854. 
28. Raymond DP, Pelletier SJ, Crabtree TD, Evans HL, Pruett TL, Sawyer RG: Impact of antibiotic-resistant Gram-negative bacilli infections on outcome in hospitalized patients. Crit Care Med 2003, 31(4):1035-1041.

29. Ezechi OC, Edet A, Akinlade H, Gab-Okafor CV, Herbertson E: Incidence and risk factors for caesarean wound infection in Lagos Nigeria. BMC Res Notes 2009, 2(1):186.

30. Cardoso Del Monte MC, Pinto Neto AM: Postdischarge surveillance following cesarean section: the incidence of surgical site infection and associated factors. Am J Infect Control 2010, 38(6):467-472.

31. Tang R, Chen HH, Wang YL, Changchien CR, Chen J-S, Hsu K-C, Chiang J-M, Wang J-Y: Risk factors for surgical site infection after elective resection of the colon and rectum: a single-center prospective study of 2,809 consecutive patients. Ann Surg 2001, 234(2):181.

32. Owens C, Stoessel K: Surgical site infections: epidemiology, microbiology and prevention. J Hosp Infect 2008, 70:3-10.

33. Classen DC, Evans RS, Pestotnik SL, Horn SD, Menlove RL, Burke JP: The timing of prophylactic administration of antibiotics and the risk of surgical-wound infection. N Engl J Med 1992, 326(5):281-286.

34. Dale WB, Peter MH: Antimicrobial prophylaxis for surgery: an advisory statement from the National Surgical Infection Prevention Project. Clin Infect Dis 2004, 38(12):1706-1715.

35. Leong G, Wilson J, Charlett A: Duration of operation as a risk factor for surgical site infection: comparison of English and US data. J Hosp Infect 2006, 63(3):255-262.

36. Campbell DA Jr, Henderson WG, Englesbe MJ, Hall BL, O'Reilly M, Bratzler D, Dellinger EP, Neumayer L, Bass BL, Hutter MM: Surgical site infection prevention: The importance of operative duration and blood transfusion -results of the first American College of Surgeons-National Surgical Quality Improvement Program Best Practices Initiative. J Am Coll Surg 2008, 207(6):810-820.

37. McGarry SA, Engemann JJ, Schmader K, Sexton DJ, Kaye KS: Surgical-site infection due to Staphylococcus aureus among elderly patients: mortality, duration of hospitalization, and cost. Infect Control Hosp Epidemiol 2004, 25(6):461-467.

38. Biadglegne F, Abera B, Alem A, Anagaw B: Bacterial isolates from wound infection and their antimicrobial susceptibility pattern in Felege Hiwot Referral Hospital, North West Ethiopia. Ethiop J health Sci 2009, 19:173-177.

doi:10.1186/2047-2994-3-25

Cite this article as: Mpogoro et al:: Incidence and predictors of surgical site infections following caesarean sections at Bugando Medical Centre, Mwanza, Tanzania. Antimicrobial Resistance and Infection Control 2014 3:25.

\section{Submit your next manuscript to BioMed Central and take full advantage of:}

- Convenient online submission

- Thorough peer review

- No space constraints or color figure charges

- Immediate publication on acceptance

- Inclusion in PubMed, CAS, Scopus and Google Scholar

- Research which is freely available for redistribution

Submit your manuscript at www.biomedcentral.com/submit 